\title{
Optimal intake of clear liquids during preparation for afternoon colonoscopy with low-volume polyethylene glycol plus ascorbic acid
}

\section{(ㄷ)(i) $\odot$}

\author{
Authors \\ Masahiro Tajika', Tsutomu Tanaka', Makoto Ishihara', Yutaka Hirayama', Sachiyo Oonishi' ${ }^{1}$, Nobumasa Mizuno², \\ Kazuo Hara², Susumu Hijioka², Hiroshi Imaoka², Toshihisa Fujiyoshi², Nobuhiro Hieda², Nozomi Okuno², Tsukasa \\ Yoshida², Kenji Yamao², Vikram Bhatia $^{3}$, Masahiko Ando ${ }^{4}$, Yasumasa Niwa' ${ }^{1}$
}

Institutions

1 Department of Endoscopy, Aichi Cancer Center Hospital, Nagoya, Japan

2 Department of Gastroenterology, Aichi Cancer Center Hospital, Nagoya, Japan

3 Department of Hepatology, Institute of Liver and Biliary Sciences, New Delhi, India

4 Center for Advanced Medicine and Clinical Research, Nagoya University Hospital, Nagoya, Japan

submitted 13.9.2016

accepted after revision 2.3.2017

\author{
Bibliography \\ DOI https://doi.org/10.1055/s-0043-106185 | \\ Endoscopy International Open 2017; 05: E416-E423 \\ (c) Georg Thieme Verlag KG Stuttgart · New York \\ ISSN 2364-3722
}

Corresponding author

Masahiro Tajika, MD, PhD , Department of Endoscopy, Aichi

Cancer Center Hospital, 1-1 Kanokoden, Chikusa-ku,

Nagoya 464-8681, Japan

Fax: +81-52-7635233

mtajika@aichi-cc.jp

\section{ABSTRACT}

Background and study aims The standard colonoscopy preparation regimen in Japan for afternoon procedures is sequential intake of $1 \mathrm{~L}$ of polyethylene glycol electrolyte lavage solution containing ascorbic acid (PEG-ASC), $0.5 \mathrm{~L}$ of clear liquid, $0.5 \mathrm{~L}$ of PEG-ASC, and finally $0.25 \mathrm{~L}$ of clear fluids (all at a rate of $0.25 \mathrm{~L}$ every $15 \mathrm{~min}$ ). However, this regimen seems poorly tolerated and complicated for many patients compared to previous regimen of polyethylene glycol electrolyte lavage solution. The aim of this study was to evaluate an alternate regimen of $0.5 \mathrm{~L}$ of PEG-ASC followed by $0.25 \mathrm{~L}$ clear liquids, repeated 3 times.

Patients and methods This was a single-blinded, non-inferiority, randomized controlled study. Subjects were randomized to the standard regimen or the alternate regimen using a web-based registry system. All patients were instructed to eat a pre-packaged, low residue diet and to take sodium picosulfate hydrate the day before colonoscopy. The Boston Bowel Preparation Scale was used to evaluate bowel cleansing, and a 3-point scale was used to assess mucosal visibility. The primary endpoint was successful bowel cleansing. The acceptability, tolerability, safety, and endoscopic findings of these two regimens were secondary endpoints.

Results A total of 409 patients were randomized to either the standard regimen $(n=204$, males $54.0 \%$, mean age 65.5 years) or the alternate regimen $(n=205,54.6 \%, 65.0$ years). The rates of successful bowel cleansing were $71.1 \%$ $(64.3-77.2 \%)$ with the standard regimen vs. $75.1 \%$ (68.6$80.9 \%$ ) with the alternate regimen ( $95 \%$ lower confidence limit, for the difference $=-4.6$, non-inferiority $P<0.05$ ). No significant differences were found in tolerability, safety, and endoscopic findings.

Conclusion The alternate regimen and standard regimen are clinically equivalent with respect to cleansing efficacy and acceptability, tolerability, safety, and endoscopic findings. These results are good news for patients with difficulty drinking the first liter of PEG-ASC.

\section{Introduction}

Adequate bowel preparation is essential for detection of colonic polyps and colorectal cancer with high sensitivity. Inadequate bowel preparation can result in failed detection of premalignant neoplastic lesions, and an increased risk of procedural ad- verse events [1,2]. Polyethylene glycol electrolyte lavage solution (PEG-ELS) is widely used for bowel preparation before colonoscopy. The efficacy of PEG-ELS is well established [3-5]. However, the poor palatability and tolerance of the $4 \mathrm{~L}$ volume of PEG-ELS reduces patient compliance with preparation [6,7]. Adjunct therapies such as bisacodyl [7,8], split regimens [9- 
12] and reduced-volume regimens [13-24] have been advocated, with better acceptability than the standard dose of PEG-ELS, without loss of efficacy. There is now an increasing preference for the use of low-volume preparations, with the 2-L PEG-ELS containing ascorbic acid becoming a market leader in Western countries. Recently, a new low-volume hyperosmolar PEG-ELS (PEG-ASC, Moviprep ${ }^{\circledR}$, EA Pharma Co., Ltd., Tokyo, Japan) has also become available in Japan.

The addition of ascorbic acid reduces the volume of the lavage solution from $4 \mathrm{~L}$ to $2 \mathrm{~L}$. Ascorbic acid itself can act as a cathartic because its absorption mechanism saturates at a high dose [25-26]. Excess ascorbic acid that cannot be absorbed remains in the bowel where it exerts an osmotic effect, acting synergistically with PEG-ELS. The addition of ascorbic acid also appears to improve the taste of the PEG-ELS preparation. However, the sequential intake of the PEG-ACS seems to be complicated compared to previous PEG-ELS regimen. In Western countries, PEG-ASC has been available for bowel preparation for colonoscopy since 2006, and many studies have demonstrated the efficacy, acceptability, tolerability, and safety of PEG-ASC compared with several standard PEG-ELS regimens [13-24].

In Japan, the use of PEG-ASC is only permitted for same-day colonoscopy. Patients are instructed to take PEG-ASC in the morning on the day of colonoscopy. As opposed to the west, patients preparing with PEG-ASC in Japan are often not advised any dietary restriction the day before the colonoscopy. However, many other hospitals including ours, instruct patients to take low residue diet and/or some laxatives to improve cleansing efficacy in practice. Although the dosing and timing of cleansing regimens including PEG-ASC have been well studied in Western countries [9, 15, 27-29], the order of PEG-ASC and clear fluid has never been studied. Therefore, the present study evaluated an alternate regimen of $0.5 \mathrm{~L}$ of PEG-ASC followed by $0.25 \mathrm{~L}$ of clear liquids, repeated 3 times, and compared it to the standard regimen under the same condition with low-residue diet and sodium picosulfate hydrate the day before colonoscopy.

\section{Patients and methods}

This was a prospective, randomized, controlled, single-center, investigator-blinded, non-inferiority study comparing the standard regimen of $1.5 \mathrm{~L}$ of PEG-ASC with an alternate regimen of $1.5 \mathrm{~L}$ of PEG-ASC in patients who underwent colonoscopy. All patients provided written, informed consent. The study was conducted at Aichi Cancer Center Hospital (ACCH), Nagoya, from April 2014 to December 2015. The study was approved by the ethics committee of ACCH, and all patients signed an informed consent form. This trial was registered in an international clinical trial registry (UMIN000014766). All consecutive outpatients of both sexes aged 20 years and older who were scheduled for an afternoon colonoscopy at ACCH were evaluated for inclusion in the study. Patients with the following clinical features were excluded: significant cardiac, renal, hepatic, or metabolic comorbidities, ascites, severe constipation (<2 bowel movements per week), known allergy to PEG-ELS, history of gastric stapling or bypass procedure, or history of prior colonic or rectal surgery. Patients were excluded if there was a suspected diagnosis of intestinal obstruction because of advanced colorectal cancer.

\section{Randomization and blinding}

Patients were randomly allocated to receive 1 of 2 different bowel preparation regimens using a computer-generated, random-number list. Randomization was performed using the Pocock and Simon minimization method [30] of balancing age ( $<70$ years or $\geq 70$ years) and sex. Concealed allocation was accomplished independently through a web-based registry system that is managed by the Data Center of Nagoya University Hospital, Nagoya, Japan. Research assistants who were not involved in this study used this web-based registry system to randomize patients.

Patients were instructed not to discuss their bowel preparation with anyone other than the unblinded research assistant. All other individuals participating in this study, including endoscopists and endoscopy nurses, were blinded to the allocated treatment group.Comparisons between the standard group and the alternate group were made in an investigatorblinded fashion.

\section{Bowel preparation methods}

The day before colonoscopy, all patients were instructed to eat a pre-packaged, low residue diet (Enimaclin CS; Horii Pharmaceutical Ind., Ltd., Osaka, Japan) that consisted of a lunch, snack, and dinner, and asked to drink more than $1 \mathrm{~L}$ of clear liquid ( $\triangleright$ Fig. 1). In the evening (at 21:00-22:00 pm) before the day of the colonoscopy, all patients were instructed to take $1 \mathrm{~mL}(7.5 \mathrm{mg})$ sodium picosulfate hydrate (Laxoberon ${ }^{\circledR}$ Solution $0.75 \%$ : Teijin Pharma, Ltd., Tokyo, Japan). On the day of the colonoscopy, all participants reported at the endoscopy room at 9:00 am or 11:00 am, and were allocated to the treatment group by using the web registry system. All patients received the bowel preparation in-hospital. In-hospital preparation was important to ensure uniformity and remove any confounding caused by poor patient adherence. More than 10 toilet facilities were made available in the endoscopy unit for patient comfort. On the day of the colonoscopy, participants received one of the two PEG-ASC (Moviprep ${ }^{\circledR}$ : each liter contained $100.0 \mathrm{~g}$ macrogol $4000,7.5 \mathrm{~g}$ sodium sulfate, $2.7 \mathrm{~g}$ sodium chloride, $1.0 \mathrm{~g}$ potassium chloride, $4.7 \mathrm{~g}$ ascorbic acid, $5.9 \mathrm{~g}$ sodium ascorbate, and lemon flavoring) regimens. The standard group was instructed to begin drinking the first $1 \mathrm{~L}$ of cleansing solution followed by $0.5 \mathrm{~L}$ of clear fluids; after that, they were instructed to begin drinking the remaining $0.5 \mathrm{~L}$ of cleansing solution followed by $0.25 \mathrm{~L}$ of clear fluids at a rate of $0.25 \mathrm{~L}$ every $15 \mathrm{~min}$. The alternate group was instructed to take $0.5 \mathrm{~L}$ of PEG-ASC followed by $0.25 \mathrm{~L}$ of clear liquids, repeated 3 times. All patients were instructed to take clear liquids after they finished drinking the cleansing solution. Colonoscopies were scheduled after 13:30 pm. 


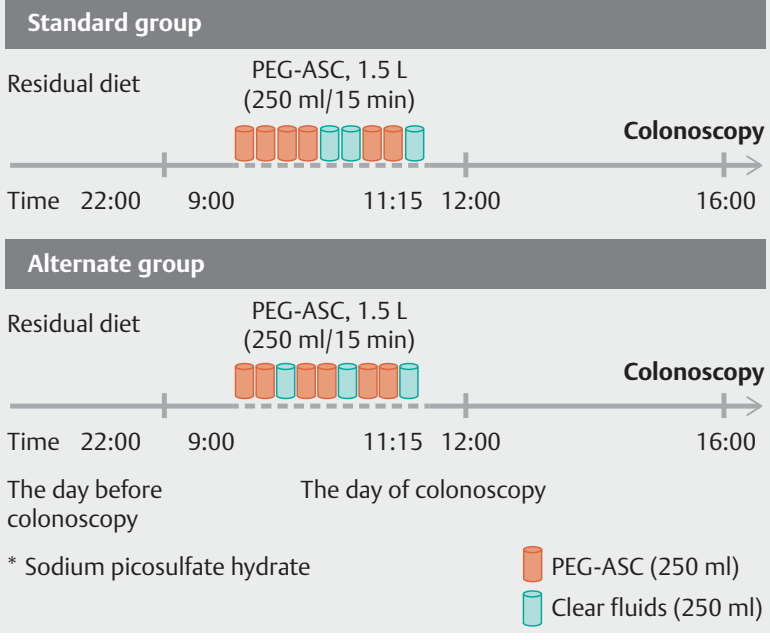

- Fig. 1 The study schedule. PEG-ELS, polyethylene glycol electrolyte lavage solution; PEG-ASC, polyethylene glycol electrolyte lavage solution containing ascorbic acid.

\section{Evaluation of bowel preparation}

Efficacy of bowel preparation was assessed using the Boston Bowel Preparation Scale (BBPS) [31]. The preparation efficacy was evaluated by the blinded endoscopist per colonic segment (right, transverse, and left colon) on a 4-point scale $(0-3)$ according to the BBPS. In addition, overall cleansing of the colon was scored by summing up the scores of each segment. For the study, the total score for each patient, ranging from 0 to 9 , was divided into 4 different classes: excellent cleansing (total score $8-9)$, good cleansing $(6-7)$, poor cleansing $(3-5)$, and inadequate cleansing (i. e., requiring additional treatment, $0-2$ ). The participating endoscopists were trained to use the BBPS scale to achieve a good level of agreement. Final assessment of the bowel preparation was summarized in 2 categories: successful or failed. A bowel preparation rated as excellent or good based on the BBPS was considered "successful," and poor or inadequate ratings were considered "failed." The investigators performed calibration exercises involving more than 20 colonoscopies prior to study commencement, based on their interpretation of scale anchors, to ensure that their findings agreed.

The physicians were also asked to score the overall mucosal visibility according to the following 3-grade scale [32]: optimal (grade 0 , clear imaging with no or a minimal amount of bubbles or foam that could be easily removed); adequate (grade 1 , modest amount of bubbles and foam that could be cleared with a minimal amount of time); or insufficient (grade 2, presence of foam and bubbles that significantly reduced the clear visualization of the mucosa). The overall mucosal visibility was assessed with washing operation before evaluation of BBPS.

During or immediately following the colonoscopy, the investigator completed a physician questionnaire regarding assessment of the bowel preparation, amount of irrigation fluid used, time needed to reach the cecum, ease of insertion into the cecum, and difficulty in observing the colorectal lumen because of peristalsis.

\section{Patient acceptability, tolerability, and other measurements}

The research assistant recorded the time required to drink the indicated volume of lavage solution. He or she also recorded the time and number of bowel movements from the start of ingestion to the appearance of clear excretion. Until 1 hour after finishing the preparation procedure, the nursing staff checked excretions. If there was a solid stool with muddy excretions or no excretion at that time, the patient was given an additional preparation, such as additional PEG-ELS or enemas. Patients who received an additional preparation were defined by the BBPS as inadequate. The patient questionnaire consisted of 20 questions. Tolerability assessment was based on the recording of gastrointestinal symptoms such as nausea, vomiting, bloating, and abdominal pain. These events were scored on a 4-point scale: $1=$ none, 2 =mild, 3 =moderate, and 4 =severe. The acceptability assessment was based on the willingness to repeat the same preparation regimen. The patients completed the questionnaire form before undergoing colonoscopy, and submitted it to the research assistant or nursing staff.

\section{End points}

The primary end point was demonstration of non-inferiority of the alternate regimen compared to the standard regimen with respect to the rate of successful bowel cleansing. Secondary end points included tolerability, acceptability, the rate of optimal mucosa visibility (score 0 ), the adenoma detection rate, and the total time for colonic preparation.

\section{Statistical analysis}

Based on a previous study [33], the rate of successful bowel cleansing of the standard regimen was expected to be $86.7 \%$. For an adequate rate, it was expected that about $86.7 \%$ of the alternate group would give a rating of successful bowel cleansing, and the non-inferiority margin was set at $-10 \%$. This study was designed to have $80 \%$ power to establish non-inferiority (using a one-sided significance level of 0.025 and a target sample size of 182). Considering an expected drop-out rate of $10 \%$, 200 patients were recruited to each group. The primary analysis for non-inferiority was performed on the per protocol (PP) population.

Baseline characteristics were summarized by the usual descriptive statistics, such as the means and standard deviation for continuous variables and rates for categorical variables. The two-sided $t$-test was used to compare the means of continuous variables; the likelihood ratio chi-squared test was used to compare the categorical measures. In addition, the Mantel extension test adjusted for age and sex was applied to compare clinical outcomes between the treatment groups.

Categorical variables were tested using the Mantel extension test. $\mathrm{P}$ values less than 0.05 were considered significant. All analyses were performed using SAS version 9.4 (SAS Institute, Cary, NC) at the Data Center of Nagoya University Hospital, Nagoya, Japan. 
Results

\section{Patient characteristics}

From April 2014 to December 2015, 416 patients were screened for inclusion in this study, and 409 patients were randomized into 2 groups; 7 patients were excluded because of exclusion criteria $(n=2)$ and consent withdrawal $(n=5)(\triangleright$ Fig. 2). The baseline characteristics of the patients are shown in $>\mathbf{T a}$ ble 1. There were no significant differences in age, sex, height, body weight, previous colonoscopies, or indications for colonoscopy between the 2 groups. Only the body mass index was significantly higher in the alternate group $(P=0.0159)$.

\section{Bowel cleansing efficacy and endoscopic findings}

Efficacy of bowel preparation and the endoscopic findings are shown in $>$ Table 2 . There was no significant difference in the successful bowel preparation rate between the standard group (71.1\%; $95 \% \mathrm{Cl} 64.3-77.2)$ and the alternate group (75.1\%; $68.6-90.9)$. The lower limit of the $95 \% \mathrm{Cl}$ of the difference in proportions between the standard group and the alternate group was $-4.6 \%$, which was higher than the non-inferiority margin of $-10 \%$. Forty-six patients in the standard group $(22.5$ $\%)$ and 39 patients in the alternate group (19.0\%) required additional preparation.

\section{Subjects screened $(n=416)$}

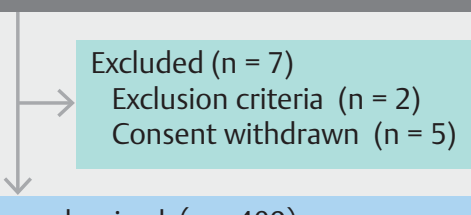

Subjects randomized $(n=409)$

Stratified by age and gender

\begin{tabular}{l|l}
$\begin{array}{l}\text { Standard group }(\mathrm{n}=204) \\
\text { Intention-to-treat analysis }\end{array}$ & $\begin{array}{l}\text { Alternate group }(\mathrm{n}=205) \\
\text { Intention-to-treat analysis }\end{array}$ \\
$\begin{array}{l}\text { Excluded due to } \\
\text { additional treatment } \\
(\mathrm{n}=43)\end{array}$ & $\begin{array}{l}\text { Excluded due to } \\
\text { additional treatment } \\
(\mathrm{n}=33)\end{array}$
\end{tabular}

Per protocol analysis $(n=161)$

Per protocol analysis $(n=172)$

Fig. 2 Patient flow.

- Table 1 Patients' characteristics.

\begin{tabular}{|c|c|c|c|}
\hline Variable & Standard group & Alternate group & $P$ value \\
\hline No. of patients & 204 & 205 & \\
\hline \multicolumn{4}{|l|}{ Age (years) } \\
\hline - mean $\pm S D$ & $65.5 \pm 11.0$ & $65.0 \pm 11.1$ & 0.6445 \\
\hline Male/female & $111 / 93$ & $112 / 93$ & 0.9640 \\
\hline Height (cm) & $161.6 \pm 8.5$ & $161.9 \pm 9.5$ & 0.7538 \\
\hline Weight (kg) & $58.5 \pm 11.0$ & $60.7 \pm 12.3$ & 0.0532 \\
\hline Body mass index $\left(\mathrm{kg} / \mathrm{m}^{2}\right)$ & 22.3 & 23.0 & 0.0159 \\
\hline \multicolumn{4}{|l|}{ History of colonoscopy, n } \\
\hline - $n>1$ & 145 & 145 & 0.9133 \\
\hline \multicolumn{4}{|l|}{ Reason for colonoscopy, n } \\
\hline - Surveillance & 95 & 101 & \\
\hline - Occult blood test-positive & 38 & 40 & \\
\hline - Screening & 30 & 24 & \\
\hline - Blood in stool & 14 & 10 & \\
\hline - Abdominal pain & 10 & 9 & 0.9013 \\
\hline - Diarrhea & 5 & 9 & \\
\hline - Constipation & 5 & 4 & \\
\hline - Anemia & 3 & 2 & \\
\hline - Other reason & 4 & 6 & \\
\hline
\end{tabular}


- Table 2 Results of preparation and endoscopic findings (Intention-to-treat analysis).

\begin{tabular}{|c|c|c|c|}
\hline Variable & Standard group & Alternate group & $P$ value \\
\hline No. of patients & 204 & 205 & \\
\hline Time to first defecation (min, mean \pm SD) & $52 \pm 22$ & $51 \pm 24$ & 0.8317 \\
\hline Frequency of defecation (times, mean \pm SD) & $9.4 \pm 3.2$ & $9.3 \pm 2.8$ & 0.6085 \\
\hline Time to preparation (min, mean \pm SD) & $170 \pm 56$ & $168 \pm 54$ & 0.6324 \\
\hline Elapsed time from last fluid intake to colonoscopy (min, mean + SD) & $116 \pm 33$ & $117 \pm 34$ & 0.8270 \\
\hline Cecal intubation rate $(n, \%)$ & $201(98.5)$ & $203(99.0)$ & 0.6527 \\
\hline Insertion time (min, mean \pm SD) & $9.4 \pm 6.0$ & $9.3 \pm 5.6$ & 0.9261 \\
\hline Feel of peristalsis $(n, \%)$ & $37(18.1)$ & $28(13.7)$ & 0.2149 \\
\hline \multicolumn{4}{|l|}{ Qualitative preparation rating (n, \%) } \\
\hline - Excellent & 101 & 99 & \\
\hline - Good & 44 & 55 & 0.6028 \\
\hline - Poor & 13 & 12 & \\
\hline - Inadequate (additional treatment) & $46(43)$ & $39(33)$ & \\
\hline Successful bowel cleansing (n, \%) & $145(71.1)$ & $154(75.1)$ & 0.3723 \\
\hline Optimal visibility grade $(0 / 1 / 2)$ & $105 / 68 / 31$ & $118 / 62 / 25$ & 0.1747 \\
\hline Adenoma detection (n, \%) & $104(51.0)$ & $116(56.6)$ & 0.2753 \\
\hline
\end{tabular}

There were no significant differences between the two groups in time to first defecation, frequency of defecation, elapsed time from last fluid intake to colonoscopy, cecal intubation rate, time needed for endoscopist to reach the cecum, and subjective difficulties in insertion to the cecum.

Efficacy rates of bowel preparation and the endoscopic findings according to per-protocol analysis are shown in $>$ Table 3. There was no significant difference in the mean total score of BBPS between the standard group $(7.7 ; 95 \% \mathrm{Cl} 7.4-7.9)$ and the alternate group $(7.6 ; 7.3-7.8)$. Successful bowel preparation rate was $90.0 \%$ for the standard group, and 89.5 for the alternate group.

\section{Patient acceptability, tolerability, and safety}

Patient tolerance and acceptance, as assessed by a questionnaire scoring subjective evaluations, are shown $>$ Table 4 . There was no significant difference in compliance between the 2 groups, taken as complete (100\%) intake of the bowel cleansing solution. The overall impression about bowel preparation of each regimen and symptoms such as nausea, vomiting, bloating, and abdominal pain was similar between the groups. Patient acceptability, that is the rate of patients who declared that they would be willing to repeat the same preparation regimen if needed, was also similar, $77.5 \%$ in the standard group compared with $75.6 \%$ in the alternate group.

\section{Discussion}

Large-volume PEG-ELS has been used worldwide since 1980 because of its proven efficacy and favorable safety profile [3-5].
However, the major limitation of its use is the volume of preparation to be ingested, which may have a negative impact on patient acceptability and compliance, resulting in reduced overall efficacy $[6,7,34]$. Current trends favor increasing use of lowvolume preparations, with $2 \mathrm{~L}$ of PEG-ASC as the market leader in Western countries. Since June 2013, PEG-ASC has been available in Japan. The efficacy, acceptability, tolerability, and safety of PEG-ASC compared with standard-volume PEG-ELS has already been demonstrated in Western countries. In Japan, a phase III trial was performed to evaluate the efficacy, acceptability, tolerability, and safety of PEG-ASC compared with standard PEG-ELS without food restriction and laxative [35]. In that study, it took $1.63 \pm 0.38 \mathrm{~L}$ of PEG-ASC to obtain the optimal colonoscopy preparation. From this result, the schedules of bowel preparation are given by the manufacturer in Japan as follows: patients are instructed to begin drinking the first $1 \mathrm{~L}$ of cleansing solution, followed by $0.5 \mathrm{~L}$ of clear fluids; after that, they are instructed to begin drinking the $0.5 \mathrm{~L}$ of cleansing solution followed by $0.25 \mathrm{~L}$ of clear fluid. In this time, if clear excretion is not confirmed, they are finally instructed to drink $0.5 \mathrm{~L}$ of PEG-ASC and $0.25 \mathrm{~L}$ of clear fluids (all at a rate of $0.25 \mathrm{~L}$ every 15 min). Compared to Western countries' regimens (e.g. patients receive $2 \mathrm{~L}$ of PEG-ASC, each liter consumed between 1 and 2 hours, and patients advised to take at least $0.5 \mathrm{~L}$ of additional clear fluid after each liter), this regimen seems poorly tolerated and complicated for many patients.

Indeed, PEG-ASC could reduce the volume of lavage solution, which might contribute to improving patient tolerance. Patients are recommended to take at least one additional liter of clear liquids with the $2 \mathrm{~L}$ of PEG-ASC solution to prevent de- 
- Table 3 Results of preparation and endoscopic findings (per protocol analysis).

\begin{tabular}{|c|c|c|c|}
\hline Variable & Standard group & Alternate group & $P$ value \\
\hline No. of patients & 161 & 172 & \\
\hline Time to first defecation (min, mean \pm SD) & $50 \pm 21$ & $49 \pm 22$ & 0.6461 \\
\hline Frequency of defecation (times, mean \pm SD) & $8.6 \pm 2.3$ & $8.8 \pm 2.4$ & 0.4599 \\
\hline Time to preparation (min, mean \pm SD) & $152 \pm 41$ & $153 \pm 40$ & 0.7918 \\
\hline Elapsed time from last fluid intake to colonoscopy (min, mean + SD) & $105 \pm 15$ & $108 \pm 15$ & 0.0869 \\
\hline Cecal intubation rate $(n, \%)$ & $156(98.7)$ & $165(99.4)$ & 0.5332 \\
\hline Insertion time (min, mean \pm SD) & $9.3 \pm 6.3$ & $9.3 \pm 5.9$ & 0.9481 \\
\hline Feel of peristalsis $(n, \%)$ & $27(17.1)$ & $19(11.4)$ & 0.1458 \\
\hline \multicolumn{4}{|l|}{ BBPS } \\
\hline - Total score (mean \pm SD) & $7.7 \pm 1.5$ & $7.6 \pm 1.5$ & 0.5878 \\
\hline - Right colon (mean \pm SD) & $2.4 \pm 0.7$ & $2.4 \pm 0.6$ & 0.7251 \\
\hline - Transverse colon (mean \pm SD) & $2.7 \pm 0.5$ & $2.6 \pm 0.6$ & 0.3212 \\
\hline - Left colon (mean $\pm S D)$ & $2.6 \pm 0.6$ & $2.6 \pm 0.6$ & 0.9520 \\
\hline Successful bowel cleansing (n, \%) & $145(90.0)$ & $154(89.5)$ & \\
\hline Optimal visibility grade $(0 / 1 / 2)$ & $82 / 53 / 23$ & $102 / 49 / 15$ & 0.0509 \\
\hline Adenoma detection (n, \%) & $78(49.4)$ & $97(58.4)$ & 0.1017 \\
\hline
\end{tabular}

- Table 4 Patient tolerance and acceptance.

\begin{tabular}{|l|l|l|}
\hline Variable & Standard group & Alternate group \\
\hline No. of patients & 204 & 205 \\
\hline $100 \%$ intake of solution (n, \%) & $193(94.6)$ & $197(96.1)$ \\
\hline $\begin{array}{l}\text { How easy/difficult to take preparation? (overall) } \\
\text { (easy/fair/difficult/very difficult) }\end{array}$ & $(71 / 99 / 21 / 13)$ & $(85 / 83 / 29 / 8)$ \\
\hline Any symptoms (n) & & \\
\hline - Nausea (none/mild/moderate/severe) & $(174 / 20 / 7 / 3)$ & $(182 / 19 / 3 / 1)$ \\
\hline - Vomiting (no/yes) & $(203 / 1)$ & $(202 / 3)$ \\
\hline - Distension (none/mild/moderate/severe) & $(68 / 79 / 43 / 14)$ & $(79 / 78 / 37 / 11)$ \\
\hline - Abdominal pain (none/mild/moderate/severe) & $(171 / 29 / 3 / 1)$ & $(170 / 31 / 4 / 0)$ \\
\hline Willingness to repeat & & 0.3039 \\
\hline - the same preparation regimen (n) (yes/no/?) & $(158 / 42 / 4)$ & 0.3657 \\
\hline
\end{tabular}

hydration, since it is slightly hyperosmolar. Although the dosing and timing of cleansing regimens have been well studied in Western countries $[9,15,27-29]$, the order of PEG-ASC and clear fluid has never been studied. Therefore, the present study evaluated an alternate regimen of $0.5 \mathrm{~L}$ of PEG-ASC followed by $0.25 \mathrm{~L}$ of clear liquids, repeated 3 times, and compared it to the standard regimen.

This study demonstrated that the alternate regimen was non inferior to the standard regimen in bowel cleansing efficacy, and it was similar to the standard regimen in patient tolerability, acceptability, safety, and endoscopic findings. To the best of our knowledge, this is the first study to evaluate the order of PEG-ASC and clear fluids for bowel preparation. The absorption of ascorbic acid reaches saturation at high doses. Thus, excess ascorbic acid, which cannot be absorbed, remains in the bowel, where it exerts an osmotic effect, acting synergistically with PEG. This is the basis for the sequential intake of differing volumes of PEG-ASC and clear liquids in the PEG-ASC regimen. 
In the present study, there was no difference in bowel cleansing efficacy between the standard group taking $1 \mathrm{~L}$ of PEG-ASC together, and the alternate group taking $1 \mathrm{~L}$ of PEG-ASC divided into 2 0.5-L bolus. With respect to bowel cleansing efficacy, there was concern that the reduction of PEG-ASC to $0.5 \mathrm{~L}$ would decrease the cathartic effects of ascorbic acid. However, there were no differences in time to first defecation, the frequency of defecation, and the time for preparation. Thus, taking $1 \mathrm{~L}$ of PEG-ASC followed by $0.5 \mathrm{~L}$ of clear liquids appears to provide the same cathartic effects of ascorbic acid as taking $0.5 \mathrm{~L}$ of PEG-ASC followed by $0.25 \mathrm{~L}$ of clear liquids, repeated twice.

We had expected patient tolerance and acceptability with the alternate regimen would improve, because taking $1 \mathrm{~L}$ of only PEG-ASC, which has a strong taste is difficult, and patients request some additional clear liquid. However, the results were similar between the 2 groups, likely because patients underwent the preparation in-hospital to maximize compliance. The patients received their bowel preparation from a nurse and research assistant who supervised its consumption, which probably provided a more accurate measure of compliance, compared to home self-preparation. Therefore, patient tolerance and acceptability with the bowel preparation solution in both groups may have been similar. Another reason may be that the taste of the solution was less unpleasant, likely due to higher concentration of ascorbic acid. However, these results are good news for patients who have difficulty taking $1 \mathrm{~L}$ of only PEG-ASC.

The key strength of this study lies in its design. To provide precise results without artificial bias as much as possible, concealed allocation was accomplished independently through a web-based registry system. Furthermore, the research assistant recorded patients' questionnaire responses and gathered physician questionnaire answers and immediately input the data into the web-based registry system.

There are a few limitations of this study. First, it was conducted in a single hospital. However, to eliminate sampling bias, a web-based registry system was used. Second, the preparation was performed in the hospital to maximize compliance, but this study design may have positively influenced patient tolerance, acceptability, and their understanding of the preparation protocol. Third, the rates of successful bowel cleansing in both groups were low compared to previous studies [13-24]. About $20 \%$ of patients in both groups needed additional treatment. One of the reasons may be that $1.5 \mathrm{~L}$ of PEG-ASC was insufficient for successful bowel cleansing, although our previous study demonstrated that use of $1.5 \mathrm{~L}$ of PEG-ASC was not inferior to use of $2.0 \mathrm{~L}$ of PEG-ELS. Another reason was that, in the present study, one hour after finishing the preparation procedure, the nursing staff checked patients' excretions, and if there was solid stool with muddy excretions or no excretion at that time, the patient was given an additional preparation. This might be an excessively strict approach. However, the high adenoma detection rate in both groups provides evidence that we achieved high quality of bowel preparation [15, 17,23]. Finally, the efficacy, acceptability, tolerability, and safety of $1.5 \mathrm{~L}$ of PEG + ASC were evaluated in a different regimen; the results may not be applicable to patients in the Western hemisphere because $2 \mathrm{~L}$ of PEG-ASC has been used there.

\section{Conclusion}

In conclusion, the alternate regimen and the standard regimen are clinically equivalent with respect to cleansing efficacy and acceptability, tolerability, safety, and endoscopic findings. These results are good news for patients with difficulty in taking $1 \mathrm{~L}$ of PEG-ASC.

\section{Acknowledgements}

The authors would like to thank all nursing staff in the Department of Endoscopy of Aichi Cancer Center Hospital and the research assistant, Mrs. Noriko Yasui.

\section{Competing interests}

This study was funded by EA Pharma Co., Ltd., Tokyo, Japan.

\section{References}

[1] Wexner SD, Beck DE, Baron TH et al. A consensus document on bowel preparation before colonoscopy: prepared by a task force from the American Society of Colon and Rectal Surgeons (ASCRS), the American Society for Gastrointestinal Endoscopy (ASGE), and the Society of American Gastrointestinal and Endoscopic Surgeons (SAGES). Gastrointest Endosc 2006; 63: 894-909

[2] Chokshi RV, Hovis CE, Hollander T et al. Prevalence of missed adenomas in patients with inadequate bowel preparation on screening colonoscopy. Gastrointest Endosc 2012; 75: 1197-1203

[3] Ell C, Fischbach W, Keller R et al. ; Hintertux Study Group. A randomized, blinded, prospective trial to compare the safety and efficacy of three bowel-cleansing solutions for colonoscopy (HSG- $01^{*}$ ). Endoscopy 2003; 35: 300-304

[4] Belsey J, Epstein O, Heresbach D. Systematic review: adverse event reports for oral sodium phosphate and polyethylene glycol. Aliment Pharmacol Ther 2009; 29: 15-28

[5] Davis GR, Santa Ana CA, Morawski SG et al. Development of a lavage solution associated with minimal water and electrolyte absorption or secretion. Gastroenterology 1980; 78: 991 - 995

[6] Aronchick CA, Lipshutz WH, Wright SH et al. A novel tableted purgative for colonoscopic preparation: efficacy and safety comparisons with Colyte and Fleet Phospho-Soda. Gastrointest Endosc 2000; 52: $346-352$

[7] Radaelli F, Meucci G, Imperiali G et al. High-dose senna compared with conventional PEG-ES lavage as bowel preparation for elective colonoscopy: a prospective, randomized, investigator-blinded trial. Am J Gastroenterol 2005; 100: 2674-2680

[8] Sharma VK, Chockalingham SK, Ugheoke EA et al. Prospective, randomized, controlled comparison of the use of polyethylene glycol electrolyte lavage solution in four-liter vs. two-liter volumes and pretreatment with either magnesium citrate or bisacodyl for colonoscopy preparation. Gastrointest Endosc 1998; 47: 167-171

[9] Marmo R, Rotondano G, Riccio G et al. Effective bowel cleansing before colonoscopy: a randomized study of split-dosage versus non-split dosage regimens of high-volume versus low-volume polyethylene glycol solutions. Gastrointest Endosc 2010; 72: 313-320 
[10] Kilgore TW, Abdinoor AA, Szary NM et al. Bowel preparation with split-dose polyethylene glycol before colonoscopy: a meta-analysis of randomized controlled trials. Gastrointest Endosc 2011; 73: 1240 1245

[11] Cohen LB. Split dosing of bowel preparations for colonoscopy: an analysis of its efficacy, safety, and tolerability. Gastrointest Endosc 2010; 72: 406-412

[12] Enestvedt BK, Tofani C, Laine LA et al. 4-Liter split-dose polyethylene glycol is superior to other bowel preparations, based on systematic review and meta-analysis. Clin Gastroenterol Hepatol 2012; 10: $1225-1231$

[13] Ell C, Fischbach W, Bronisch H] et al. Randomized trial of low-volume PEG solution versus standard PEG + electrolytes for bowel cleansing before colonoscopy. Am J Gastroenterol 2008; 103: 883-893

[14] Corporaal S, Kleibeuker JH, Koornstra JJ. Low-volume PEG plus ascorbic acid versus high-volume PEG as bowel preparation for colonoscopy. Scand J Gastroenterol 2010; 45: 1380-1386

[15] Matro R, Shnitser A, Spodik M et al. Efficacy of morning-only compared with split-dose polyethylene glycol electrolyte solution for afternoon colonoscopy: a randomized controlled single-blind study. Am J Gastroenterol 2010; 105: 1954-1961

[16] Jansen SV, Goedhard JG, Winkens B et al. Preparation before colonoscopy: a randomized controlled trial comparing different regimes. Eur ] Gastroenterol Hepatol 2011; 23: 897-902

[17] Pontone S, Angelini R, Standoli M et al. Low-volume plus ascorbic acid vs high-volume plus simethicone bowel preparation before colonoscopy. World J Gastroenterol 2011; 17: 4689-4695

[18] Valiante F, Pontone $S$, Hassan C et al. A randomized controlled trial evaluating a new 2-L PEG solution plus ascorbic acid vs 4-L PEG for bowel cleansing prior to colonoscopy. Dig Liver Dis 2012; 44: 224 227

[19] Kelly NM, Rodgers C, Patterson $N$ et al. A prospective audit of the efficacy, safety, and acceptability of low-volume polyethylene glycol $(2 \mathrm{~L})$ versus standard volume polyethylene glycol $(4 \mathrm{~L})$ versus magnesium citrate plus stimulant laxative as bowel preparation for colonoscopy. J Clin Gastroenterol 2012; 46: 595-601

[20] Ponchon T, Boustière C, Heresbach D et al. A low-volume polyethylene glycol plus ascorbate solution for bowel cleansing prior to colonoscopy: the NORMO randomised clinical trial. Dig Liver Dis 2013; 45 : $820-826$

[21] Gentile M, De Rosa M, Cestaro G et al. 2 L PEG plus ascorbic acid versus 4 L PEG plus simethicon for colonoscopy preparation: a randomized single-blind clinical trial. Surg Laparosc Endosc Percutan Tech 2013; 23: 276-280

[22] Moon CM, Park DI, Choe YG et al. Randomized trial of 2-L polyethylene glycol + ascorbic acid versus 4-L polyethylene glycol as bowel cleansing for colonoscopy in an optimal setting. J Gastroenterol Hepatol 2014; 29: $1223-1228$
[23] Rivas JM, Perez A, Hernandez M et al. Efficacy of morning-only 4 liter sulfa free polyethylene glycol vs 2 liter polyethylene glycol with ascorbic acid for afternoon colonoscopy. World J Gastroenterol 2014; 20: $10620-10627$

[24] Xie Q, Chen L, Zhao F et al. A meta-analysis of randomized controlled trials of low-volume polyethylene glycol plus ascorbic acid versus standard-volume polyethylene glycol solution as bowel preparations for colonoscopy. PLoS One 2014; 9: e9902

[25] Wilson JX. Regulation of vitamin C transport. Annu Rev Nutr 2005; 25 $105-125$

[26] Fujita I, Akagi Y, Hirano J et al. Distinct mechanisms of transport of ascorbic acid and dehydroascorbic acid in intestinal epithelial cells (IEC-6). Res Commun Mol Pathol Pharmacol 2000; 107: 219-231

[27] Eun CS, Han DS, Hyun YS et al. The timing of bowel preparation is more important than the timing of colonoscopy in determining the quality of bowel cleansing. Dig Dis Sci 2011; 56: 539- 544

[28] Siddiqui AA, Yang K, Spechler S] et al. Duration of the interval between the completion of bowel preparation and the start of colonoscopy predicts bowel-preparation quality. Gastrointest Endosc 2009; 69: $700-706$

[29] Church JM. Effectiveness of polyethylene glycol antegrade gut lavage bowel preparation for colonoscopy-timing is the key! Dis Colon Rectum 1998; 41: $1223-1225$

[30] Pocock S], Simon R. Sequential treatment assignment with balancing for prognostic factors in the controlled clinical trial. Biometrics 1975; 31: $103-115$

[31] Lai EJ, Calderwood AH, Doros G et al. The Boston bowel preparation scale: a valid and reliable instrument for colonoscopy-oriented research. Gastrointest Endosc 2009; 69: 620-625

[32] Repici A, Cestari R, Annese V et al. Randomised clinical trial: low-volume bowel preparation for colonoscopy - a comparison between two different PEG-based formulations. Aliment Pharmacol Ther 2012; 36 : $717-724$

[33] Tajika M, Tanaka T, Ishihara M et al. A Randomized Controlled Trial Evaluating a Low-Volume PEG Solution Plus Ascorbic Acid versus Standard PEG Solution in Bowel Preparation for Colonoscopy. Gastroenterol Res Pract 2015; 2015: 326581

[34] Froehlich F, Wietlisbach V, Gonvers J] et al. Impact of colonic cleansing on quality and diagnostic yield of colonoscopy: the European Panel of Appropriateness of Gastrointestinal Endoscopy European multicenter study. Gastrointest Endosc 2005; 61: 378-384

[35] Ueno F, Kudo SE, Igarashi M. Efficacy and safety of a deduced-volume polyethylene glycol electrolyte lavage solution plus ascorbic acids in preparation for colonoscopy: a randomized trial. J New Rem \& Clin 2016; 5: $681-695$ 